\title{
Adaptive Cross-layer Resource Allocation by HNN in OFDM-MISO System
}

\author{
${ }^{\text {a1 }}$ Mingyan Jiang, ${ }^{\text {a2 }}$ Yulong Liu \\ ${ }^{a}$ School of Information Science and Engineering Shandong University Jinan, 250100, China
}

\begin{abstract}
This paper presents an adaptive cross-layer resource allocation problem with the fairness in multi-user OFDMMISO communication systems, and provides two solutions with Hopfield Neural Network (HNN) and Genetic Algorithm (GA) for the problem. We utilized HNN's characteristics such as parallel processing, fast convergence speed and easy convergence to the optimum, to solve this problem under the conditions of proportional fairness for satisfying system performances and users' requirements. The method is simplified in the computation by dividing the bit-loading matrix into three matrixes. The simulation results show that HNN and GA can effectively solve optimization problems of resource allocation in such system, and results of selected HNN and GA methods are more effective than that of the traditional method.
\end{abstract}

Index Terms: Hopfield neural network; multi-user OFDM-MISO system; proportional fairness; cross-layer design; resource allocation

(C) 2012 Published by MECS Publisher. Selection and/or peer review under responsibility of the Research Association of Modern Education and Computer Science

\section{Introduction}

The authors considered the problem of scheduling and resource allocation[1] for multi-user video streaming over downlink Orthogonal Frequency Division Multiplexing (OFDM) channels and the video streams are precoded with the video coding scheme, and the authors focus on the problem of maximizing the overall spectral efficiency[2] while maintaining the QoS requirements of users, including bit error rate and individual minimum rate requirements. A novel cross-layer sub-carrier and bit allocation algorithm[3] aims to maximize the system throughput with the overall transmit power constraint and user rate proportionality constraint. Schedulerallocator architecture in a loose cross-layer strategy is proposed in order to improve the efficiency of radio resource allocation. In paper[4], the authors developed an adaptive cross-layer scheduling scheme combined with dynamic sub-carrier allocation algorithm and the proposed adaptive cross-layer scheduling and dynamic sub-carrier allocation algorithm can reduce delays and improve the spectral efficiency in Rayleigh fading channel. A complete-fairness cross layer optimization framework is proposed[5], which considers fairness for both sources and relays. 
Paper[6] proposed a novel algorithm, and used greedy algorithm to solve the problem, but the greedy algorithm is a local sub-optimal algorithm without taking into account the overall various possible situations. The objective of cross-layer formulation[7] is to allow joint determination of power control, frequencyselective OFDM scheduling and multi-hop routing in order to maximize the minimum throughput, but their approach was based on water-filling method which is very complex, thus not practical. In paper[8], HNN has been proven to be successful in solving dynamic resources allocation problems in a short time, but the crosslayer optimization was not taken into account. According to optimal or sub-optimal system capacity schemes, authors [9] proposed intelligent algorithm selected a user combination for a fixed number of users within few generation iterations, but it did not combine OFDM and Multiple-Input Single-Output (MISO) as one system leading to the question too simple.

From above, we propose the HNN and GA methods for the problem which is cross-layer resource allocation optimization problem with the fairness in multi-user OFDM-MISO System.

\section{Model and Analysis}

First, Assume that a system with a base station equipped with $N_{T}$ antennas and $K$ mobile users, and each user equipped with one antenna is receiving signals from the base station. Considering information bits allocated on the $m$ th sub-carrier from the $K$ users are first encoded to produce the encoded symbols as $U_{m, 1}, \cdots, U_{m, k} \rightarrow$.The encoded symbols are assumed to have unit variance and are amplified by the respective

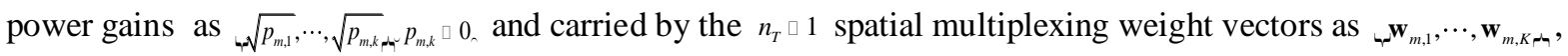
respectively. The processed signals are fed to the $m$ th inputs in the bank of $n_{T}$ Inverse-Discrete-FourierTransform (IDFT) processors, and the $n_{T} \square n_{f}$ outputs of the IDFT are first added with cyclic prefix. The resulting $n_{T} \square n_{f} \square N_{\text {cyclic }}$ blocks of time-domain samples are transmitted over the $n_{T}$ transmit antennas column by column. At the receiver of each of the mobile users, there is a corresponding DFT block. So at the $m$ th input of the IDFT block, the $n_{T} \square 1$ transmitted signal $\mathbf{B}_{\mathbf{m}}$ is given by $\mathbf{B}_{m} \square \square_{k=1}^{K} \sqrt{p_{m, k}} \mathbf{w}_{m, k} U_{m, k}$. Without loss of generality, the $n_{T} \square 1$ frequency-domain received signal vector of the $k$ th mobile user is given by $\mathbf{Y}_{k} \square \mathbf{H}_{k} \mathbf{B} \square \mathbf{Z}_{k}$, where $\mathbf{B}$ is the $n_{T} n_{f} \square 1$ aggregate transmit vector at the input of IDFT, given by $\mathbf{B}_{\square}$ diagonal channel matrix aggregated over the $n_{f}$ sub-carriers of user $k$, given by $\begin{array}{ccc}\mathbf{H}_{k} & \begin{array}{c}\mathbf{H}_{0, k} \\ 0\end{array} & 0 \\ 0 & 0 & 0\end{array}$ denotes the $1 \square n_{T}$ channel matrix between the $n_{T}$ transmit antennas and the $k$ th mobile user in frequency domain and $\mathbf{Z}_{k}$ presents the $n_{f} \square 1$ zero-mean white Gaussian noise with covariance matrix ${ }_{-2}^{2}$.

Hence, considering $m$ th sub-carrier allocated on the $k$ th mobile user, the received signal is given by $\mathbf{Y}_{m, k} \square \mathbf{H}_{m, k} \mathbf{B}_{m} \square \mathbf{Z}_{m, k}$

$\left.\square \sqrt{p_{m, k}} H_{m, k} \mathbf{w}_{m, k} U_{m, k} \square\right] \sqrt{p_{m, j}} \mathbf{H}_{m, j} \mathbf{w}_{m, j} U_{m, j} \square \mathbf{Z}_{m, k}$

Similarly, we shall adopt the zero-forcing (ZF) approach in choosing the spatial multiplexing weights $\downarrow_{r} \mathbf{w}_{m, k+\hookrightarrow}$. Define $A_{m} \square, k \square\left[1, K \square: p_{m, k} \triangleright 0_{\lrcorner \downarrow}\right.$ : as the set of active users corresponding to the $m$ th sub-carrier. 


\section{Cross-layer Design for OFDM-MISO}

In the OFDM-MISO system, sub-carriers can be allocated on a user depending on the instantaneous channelstate-information (CSI). So the cross layer scheduling algorithm can be cast into the following optimization problem. Choose the user's set allocation $A_{\square} A_{0}, \cdots, A_{n f \_}$sc and the power allocation $p \square p_{m, k}: m \square \boxminus 0, n_{f} \square 1 \square, k \square A_{m \downarrow \downarrow}$ according to the given CSI realization $\mathbf{H}_{1}, \cdots, \mathbf{H}_{K}$, under the conditions of $n_{f}-1$ $\square \square m_{m=0} p_{k \in A_{m}} \square p_{0}$ and $\left|A_{m}\right| \square n_{T}$ for all $m \square \boxminus 0, n_{f} \square 1 \boxminus$. Assume that the total transmission power from the base station is constrained at $P_{0}$. The objective is to optimize the maximization of the system throughput $\left.\square{ }_{k=1}^{K} r_{k}\right\lrcorner \mathbf{H}_{\curvearrowleft}$, where $r_{k}$ is given by equation:

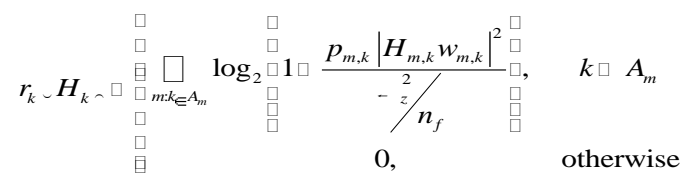

\section{Steps of Cross-layer Design Algorithm}

There are two sets of optimization variables: the active user allocation $\mathbf{A}$ and the power allocation $\mathbf{P}$. The former is a discrete variable and the latter is a continuous variable. In our approach algorithm, the sub-carrier with the highest channel gain among the available sub-carriers should be allocated to a node to achieve the best throughput performance. Therefore, the optimization problem can be solved in the following steps.

Step 1: Initialization. For each sub-carrier $m \square \square 0, n_{f} \square \mathbf{1}_{\square}$, initialize the active user sets $\mathbf{A}_{m}$ such that $\left|\mathbf{A}_{m}\right| \square n_{T}$.

Step 2: Optimization with respect to $\mathbf{A}$. Let $\cdots_{-1}, \cdots,,_{k}$, be a discrete decision variable to represent $\mathbf{A}$. If subcarrier $n$ is allocated to use $k,{ }_{-} k, n$ $\square$, otherwise ${ }_{-k, n} \square 0$.

Step 3: Optimization with respect to $\mathbf{P}$. Here we adopt M-QAM modulation, when adopting HNN to solve the

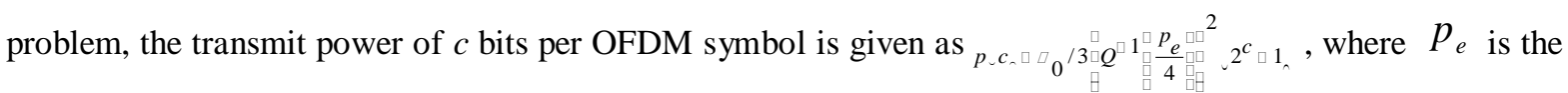
required bit-error-rate (BER), and Q-function is defined as $Q_{\lrcorner x} \frac{1}{\sqrt{2}} \square e^{\square} t^{2 / 2} d t$, so from bit matrix $\mathbf{C}_{\text {we can }}$ calculate the power allocation $\mathbf{P}$. When we adopt GA to solve the problem, given the active user sets $\mathbf{A}_{m}$, the optimization problem can be written into the following Lagrangian function:

$$
L_{\sim} p_{m, k}, \ell, \square \square_{m=0}^{n_{f}-1} \square \square \cos _{k \in} \square \log _{2} \square 1 \square \frac{p_{m, k}\left|H_{m, k} w_{m, k}\right|}{-{ }_{z}^{2} / n_{f}} \square \ell p_{m, k} \square
$$

where $\ell$ is the Lagrange multiplier chosen to satisfy the total transmit power constraint $P_{0}$. With standard optimization techniques and the Karush-Kuhn-Tucker (KKT) condition, the optimal power allocation is given by

$$
p_{m, k}^{*} \underset{\square}{\square} \frac{1}{\ln 2 \Omega \ell} \square \frac{-{ }_{z}^{2} / n_{f}}{\left|H_{m, k} w_{m, k}\right|^{2}}{ }^{+}
$$




\section{Optimization Using the GA Method}

The main optimization steps with GA for the problem are described as follows:

(1).Coding: Basic features of genetic algorithm are that they work on coding space and solution space alternatively.

(2).Crossover: Crossover Operates on two chromosomes at a time and generates offspring by combining both chromosomes' features. For two chromosomes chosen with crossover possibility $p_{c}$, we use arithmetical crossover operators.

(3).Fitness function: The particular chromosome can be ranked against all the other chromosomes. fitness function is defined in (2).

(4).Mutation: For each chromosome chosen with mutation probability $p_{m}$, the method replaces each element with a random value.

(5).Optimization process:

Step1: Initialize population. Each element in the chromosome is a power allocated to sub-carriers, thus, population is a 2-D array, where the rows represent chromosome numbers and columns of a row represent subcarriers.

Step2: Evaluate the individual values in population using fitness function.

Step3: Generate the new population using crossover and mutation probability.

Step4: Repeat step2 and step3 till the algorithm converges.

\section{Optimization Using the HNN Method}

\section{1 User set allocation}

The optimization problem is reformulated as:

$R \square \square_{m=1}^{M} \square_{k=1}^{K}-\log _{2, k}^{\square} \log _{\square} \frac{P\left|H_{m, k} w_{m, k}\right|^{2} \square}{-{ }_{z}^{2} / n_{f}} \square$

Subject to :

(i) $1 \square \underset{k \square 1}{K}-k, n, n \square \downarrow l, 2, \cdots, N_{\lrcorner}$, it denotes that the sub-carrier is allocated on each user.

(ii) $n_{k} \underset{n}{\quad} \underset{n \square 1}{N}-k, n$, it denotes the fairness constraint

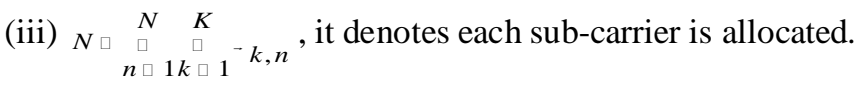

(iv) $R_{i}: R_{j} \square \mathfrak{\downarrow}_{i}: \mathfrak{\downarrow}_{j}$, it denotes the initial rate of each node constraint.

For the sub-carrier allocation, the energy function proposed by HNN is:

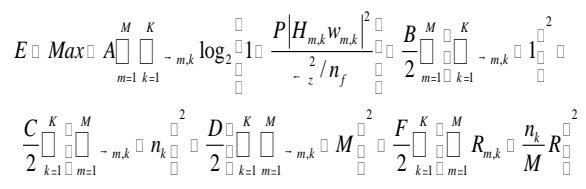

The dynamic equation of the HNN method can be calculated as: 


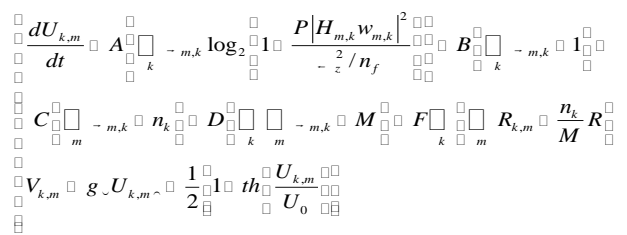

From above, we structure the HNN for solving the user set allocation. By the networks, from the initial state $U_{0}$, after the continuously iterated by the dynamic equation of the networks, we obtain the output of the networks which is the user set allocation matrix $\rho$.

\section{2 Bit-loading}

In order to solve the problem, we divide bit matrix $\mathbf{c}$ into three matrixes: sub-carrier allocation matrix $\boldsymbol{\rho}$, HNN output matrix $\mathbf{v}, v_{n, j} \square_{\downarrow}, 1_{\lrcorner \_}$for all $n, j$, and bit allocation matrix I whose elements are denoted the number of bits allocated on the every sub-carrier, $l_{j, n} \square, 0,1, \cdots, M_{\lrcorner}$. We give the method of calculating $\mathbf{c}$ as follows:

1) The elements of matrix $\mathbf{v}$ are multiplied by the corresponding of the matrix $\mathbf{l}$, then get matrix npn ;

2) Matrix npn is multiplied by matrix $\mathbf{k p n}_{1 \square j}$. whose elements are all $1 \mathrm{~s}$, then get matrix mpn .

3) Extending matrix mpn to k lines, get matrix lpn, and then the elements of matrix lpn are multiplied by the corresponding of the matrix $\boldsymbol{\rho}$, at last we get matrix $\mathbf{c}$.

The optimization problem is formulated as:

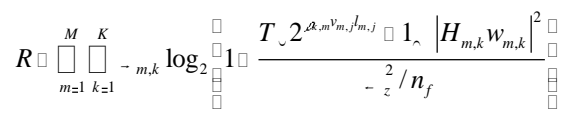

where $T \square \frac{N_{0}}{3} \square Q^{-1} \square \frac{p_{e}}{4} \square \square^{2}$

For the bit-loading, the energy function proposed by the HNN method is:

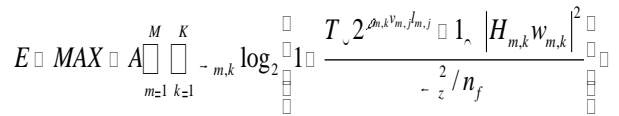

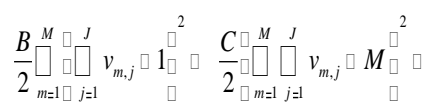

$\frac{D}{2} \square_{k=1 \square m=1}^{K} \square R_{k, m}^{M} \square \frac{n_{k}}{M} R_{\square}^{\square} \square \frac{F \square \square}{2} \square \square_{\square=1}^{N} p_{m=1}^{J} \square P_{\text {total }} \square$

1) The first term of the energy function enforces the $\mathrm{HNN}$ algorithm to maximize the allocated bit rates.

2) The second term ensures that the number of bits allocated on each sub-carrier is certain.

3) The third term ensures that each sub-carrier is used.

4) The forth term denotes the rate of each node constraint.

5) The sixth term denotes the total power constraint.

For the above problem, the dynamic equation of the HNN method can be calculated as: 


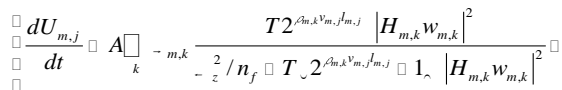

$$
\begin{aligned}
& \square B_{\square}^{\square} v_{m, j} \square 1_{\square}^{\square} C_{\square}^{\square} \square v_{j} v_{m, j} \square M_{\square \square}^{\square} \\
& \square \square \underset{k \square m-1}{\square} R_{k, m} \square \frac{n_{k}}{M} R_{\square}^{\square} F_{\square}^{\square} \square \underset{k}{\square} p_{m, k} \square P_{\text {total }}^{\square} \square \\
& V_{m, j} \square g_{\checkmark} U_{m, j} \square \frac{1}{2} \square{ }_{\square} \square t h_{\square}^{\square} \frac{U_{m, j}}{U_{0}} \square \square
\end{aligned}
$$

From above, we structure the HNN to solve the bit allocation, and obtain the HNN output matrix $\mathbf{v}$, then get bit-loading matrix $\mathbf{c}$.

In order to ensure $E \square 0$, we add a constant $M A X$, constants $A, B, C, D$, and $F$ are the weigh terms of the energy function and the dynamic equation, and their values are selected to obtain a fast convergence of the desired solution.

\section{Parameter Setting and Simulation Result}

We do simulation in OFDM/MISO system with 10 transmitting antennas, 10 mobile users, modulation is 64QAM. The wireless channel is modeled as a frequency-selective channel consisting of 12 independent Rayleigh-multipaths. White noise ${ }_{-}{ }^{2} \square 10^{-4}$; Bit error rate $B E R \square 10^{-4}$. HNN' parameters setting: $A=100, B=500$, $C=50, D=10, F=10$;

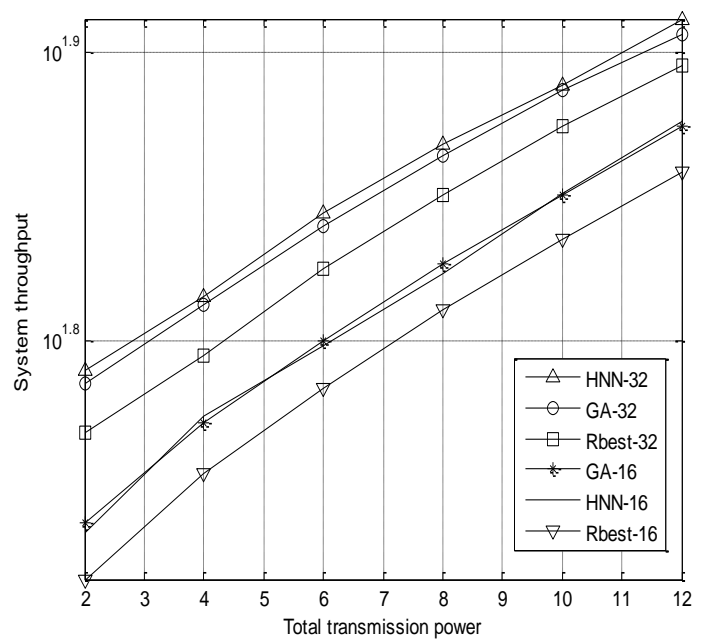

Figure 1.Performance comparison of the throughputs

HNN neurons slope is $U_{0}=0.02$, HNN calculation step is 0.0001 , the times of iteration is 2000 . The parameters of GA for the problem are provided below:

We choose the linear structure coding method, through which a chromosome presents a feasible solution of the power allocation; Population size $=20$; Terminal generation number $=100$; Crossover probability $=0.6$; Mutation probability $=0.1$.

Fig. 1 plots the throughputs respectively when the number of sub-carriers is 16 or 32 . HNN-16, HNN-32 denoted the throughputs when the number of sub-carriers is 16 or 32 adopted by HNN algorithm under the different transmit power constraints; GA-16, GA-32 denoted the throughputs adopted by GA algorithm; and 
Rbest-16, Rbest-32 denoted the throughputs when it did not take into account the multi-users option. As is known, in OFDM system as the users' number increase, the increase of system diversity gain can lead to the increase of system throughput, which is consistent with the simulation results, as shown in Fig.1. Clearly, the general effectiveness based on HNN and GA algorithm is superior to that adopted by Rbest's algorithm[10], because system resources can be more reasonable distribution after the multi-users selection. HNN and GA algorithm can effectively solve the problem as a global optimization algorithm, but the effect of the former is better than that of the latter when the number of sub-carrier is 32 as shown in the figure, which indicates that HNN algorithm is more suitable to solve this problem in large-scale systems. However, with the increase of users' number, the number of system's local sub-optimal solutions sharply increases, which leading to performance degradation of HNN and GA.

Generally, the values of parameters A, B, C, D, F range from 0 to 1000, and their values are not the same when we solve different problems; But their values have a major impact on the effectiveness and level of the network convergence; After several simulations, we obtain that parameters' values are inversely proportional to the convergence value of the corresponding items in dynamic equations.

\section{Conclusion}

In this paper, we propose the HNN and GA methods for the problem which is cross-layer resource allocation optimization problem with the fairness in multi-user OFDM-MISO system. At the same time we divide the bitloading matrix into three matrixes, which can simplify the problem in such system. Our approach is to decompose the problem into two steps: user set allocation and bit-loading, by taking HNN's and GA's advantages of the inherent parallelism, a real-time running and its global optimum. The simulation results show that our proposed methods by HNN and GA can effectively solve optimization problems in OFDM-MISO system, and the general effectiveness based on HNN is superior to that adopted by GA and traditional methods.

\section{Acknowledgment}

This work is supported by the Natural Science Foundation of Shandong Province (No. ZR2010FM040 ).

\section{References}

[1] Xin Ji, Jianwei Huang, Mung Chiang, Catthoor, F.; "Downlink OFDM Scheduling and Resource Allocation for Delay Constraint SVC Streaming"; Communications, 2008. ICC '08. IEEE International Conference on 19-23 May 2008 Page(s):2512 - 2518.

[2] Wei Xu, Chunming Zhao, Peng Zhou, Yijin Yang; "Efficient Adaptive Resource Allocation for Multiuser OFDM Systems with Minimum Rate Constraints"; Communications, 2007. ICC '07. IEEE International Conference on 24-28 June 2007. pp:5126 - 5131.

[3] Todini, A.; Moretti, M.; Valletta, A.; Baiocchi, A.; "A Modular Cross-Layer Scheduling and Resource Allocation Architecture for OFDMA Systems"; Global Telecommunications Conference, 2006. GLOBECOM '06. IEEE Nov. 27 2006-Dec. 1, 2006 , pp:1 - 6.

[4] Xuebing Pei, Gan Liu, Guangxi Zhu, Li Li; "Adaptive Cross-layer Scheduling and Dynamic Subcarrier Allocation Algorithm Based on Service-differentiation in Multiuser OFDMA System”; Communications and Networking in China, 2007. CHINACOM '07. Second International Conference on 22-24 Aug. 2007, pp:1057 - 1061.

[5] Jianwei Wang; Yuping Zhao; Korhonen, T.; "Cross Layer Optimization with Complete Fairness Constraints in OFDMA Relay Networks"; Global Telecommunications Conference, 2008. IEEE GLOBECOM 2008. IEEE Nov. 30 2008-Dec. 4 2008, pp:1 - 5. 
[6] Peng Cheng, Guanding Yu, Zhaoyang Zhang, Peiliang Qiu; "A Cross-Layer Fair Resource Allocation Algorithm for OFDMA Systems"; Communications, Circuits and Systems Proceedings, 2006 International Conference, Volume 2, 25-28 June 2006, pp:1342 - 1346.

[7] Kemal Karakayali, Joseph H. Kang, Murali Kodialam, and Krishna Balachandran; "Cross-Layer Optimization for OFDMA-Based Wireless Mesh Backhaul Networks"; Wireless Communications and Networking Conference, 2007, WCNC 2007, IEEE 11-15 March 2007, pp.276 - 281.

[8] Daniel Calabuig, José F. Monserrat, David Gómez-Barquero, and Narcís Cardona; "A delay-Centric Dynamic Resource Allocation Algorithm for Wireless Communication Systems Based on HNN"; Vehicular Technology, IEEE Transactions, Volume 57, Issue 6, Nov. 2008, pp.3653 - 3665.

[9] Mingyan Jiang, Rubio F., Yong Wang, Gomez, J., Dongfeng Yuan. User Selection for Maximum Sum-rate in Multi-user and MISO System with Evolutionary Algorithm, Cross Layer Design, IWCLD '07, Sept. 2007, pp: $74-77$.

[10] Vincent L. N. Lau,Yu K-Wong Ricky Kwok. Channel-Adaptive Technologies and Cross-Layer Designs for Wireless Systems with Multiple Antennas [J], Wiley-Interscience, 2006, 44(9):28-28. 